\title{
Pasta structures in neutrino-degenerate nuclear matter
}

\section{Toshiki Maruyama*}

Japan Atomic Energy Agency, Shirakata Shirane 2-4, Tokai, Ibaraki, 319-1195 Japan

Email: maruyama.toshiki@jaea.go.jp

\section{Nobutoshi Yasutake}

Department of Physics, Chiba Institute of Technology, Narashino, Chiba, 275-0023 Japan

\section{Toshitaka Tatsumi}

Department of Physics, Kyoto University, Kyoto, 606-8502 Japan

\begin{abstract}
We explore inhomogeneous structures and properties of low-density nuclear matter in the presence of neutrinos, which are relevant to supernova cores and protoneutron stars. As a theoretical framework we employ a relativistic mean-field model with the Thomas-Fermi approximation for fermions, which is able to describe well-known bulk properties of nuclei and well-accepted properties of symmetric nuclear matter. In this framework with a soft density-dependence of symmetry energy, low-density nuclear matter without neutrinos exhibits "pasta" structures only if the proton fraction is large enough. For catalyzed nuclear matter which has small proton fraction, only spherical droplets have appeared. However, with inclusion of neutrinos by setting a constant lepton fraction, the pasta structures are found to be persistent due to the increased proton fraction. Though the structure of neutrino-trapped nuclear matter is similar to that of nuclear matter with a fixed proton fraction, the equation of state is significantly different due to the neutrino contribution to the pressure.
\end{abstract}

XII International Symposium on Nuclei in the Cosmos,

August 5-12, 2012

Cairns, Australia

* Speaker. 


\section{Introduction}

Nuclear matter is considered to be a Van der Waals fluid: It has a free-energy minimum at a certain density, i.e., the saturation density. Below the saturation density the pressure becomes negative and the system is expected to undergo a liquid-gas phase transition, which is of the firstorder. Thus nuclear matter below the saturation density generally consists of mixture of dense liquid and dilute gas. The relation between the pressure and the density, i.e., equation of state (EOS), is often obtained by applying the Maxwell construction. However, the systems where the Maxwell construction can be applied are limited to those with a single chemical component. For systems with more than one chemical component, the Maxwell construction does not satisfy the Gibbs conditions, i.e., equilibrium of chemical potentials and partial pressures of components in coexisting phases. What is characteristic of nuclear matter is that it consists of more than one chemical component and some of them have electric charge. In this case the mechanical balance as well as the chemical balance between coexisting phases should be satisfied.

Ravenhall et al. [1] and Hashimoto et al. [2] have proposed that spherical droplets of nuclei change their shapes to cylindrical rods, planar slabs, cylindrical tubes, and spherical bubbles with increasing density before melting into uniform matter. This series of non-spherical shapes is often called "pasta". Pasta structures appear as ground states of matter below the saturation density. The optimum shape and size of the structure is determined by a balance between the Coulomb repulsion and the surface tension: Roughly speaking, the Coulomb energy per baryon $E_{\mathrm{Coul}} / A$ depends on the size of the structure $R$ by a second power $E_{\mathrm{Coul}} / A \propto R^{2}$, and the surface energy per baryon $E_{\text {surf }} / A$ depends on the inverse of $R$ as $E_{\text {Coul }} / A \propto R^{-1}$. Hence the sum of both has a minimum at a

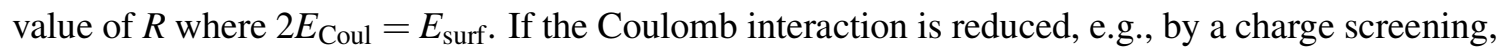
or the surface tension is enhanced, then the minimum point moves to a lager $R$. In the extreme case of above, the minimum point diverges and the structure becomes mechanically unstable, i.e., amorphous mixed phase appears [3]. Nevertheless, inhomogeneous mixed phase is expected to appear when the energy gets lower than that of uniform matter. This energy gain which should exceed the energy loss of $E_{\text {Coul }}+E_{\text {surf }}$ is achieved by the deeper binding energy of dense liquid phase which is largest at $\rho^{\text {liq }}=\rho_{0}$ and with a proton fraction $Y_{p}^{\text {liq }}=0.5$. Therefore, the structured mixed phase becomes more stable when the average baryon density is well below the saturation density $\rho_{0}$ and the proton fraction $Y_{p}$ is close to 0.5 . In fact, in our previous calculation [4] the upper limit of pasta structure is $0.11 \mathrm{fm}^{-3}$ for $Y_{p}=0.5,0.10 \mathrm{fm}^{-3}$ for $Y_{p}=0.3$, and $0.08 \mathrm{fm}^{-3}$ for $Y_{p}=0.1$.

The sites where pasta phases are expected are the crust region of neutron stars and the core of supernovae. In the former case of cooled neutron stars, the temperature is low enough to assume $T=0$ and there is no trapped neutrinos. In the case of protoneutron stars and core of supernovae, effects of finite temperature and trapped neutrinos should be taken into consideration. In the practical calculation, however, trapped neutrinos are often omitted and a fixed value of proton fraction, i.e., $Y_{p} \sim 0.3$ is assumed. By the presence of neutrinos, the proton fraction of matter in beta equilibrium significantly increases and $Y_{p}=0.3$ is a good number.

It is well known that the proton fraction of matter in beta equilibrium depends on the inhomogeneous structure. If we assume uniform matter, proton fraction $Y_{p}$ monotonically decreases with decreasing density, while $Y_{p}$ increases to approach to $\sim 0.5$ in the low-density limit. There- 
fore, the dependence of $Y_{p}$ on the density in the presence of neutrinos is not trivial. There are some preceding works by Ogasawara et al. [5, 6] and Watanabe et al. [7] on the structure and EOS of neutrino-trapped matter. It has been reported that the presence of neutrinos enhances the appearance of inhomogeneous structure of matter by increasing proton fraction. In Refs. [5, 6], only spherical nuclei and bubbles have been taken into account, and in Ref. [7] pasta structures are considered but the temperature has been limited to zero. In this paper, we explore the properties of nuclear matter at finite temperature and finite neutrino fraction taking account of pasta structures at low densities.

\section{Relativistic Mean-Field and Thomas-Fermi Approximation}

We employ a relativistic mean-field (RMF) model and the Thomas-Fermi approximation. In the RMF model, baryons (proton $p$ and neutron $n$ ) interact via the coupling with $\sigma, \omega$, and $\rho$ mesons. All the fields of baryons, mesons, and electrons $e$ are introduced in a Lorentz-invariant way. By the Thomas-Fermi approximation, the momentum distributions of fermions are assumed to have those of Fermi gas at finite temperature. Our framework is not only relatively simple for numerical calculations, but also sufficiently realistic to reproduce bulk properties of finite nuclei as well as the saturation properties of nuclear matter [4, 8]. One characteristic is that the Coulomb interaction is properly included in the equations of motion for baryons and electrons and for meson mean-fields. Thus the baryon and electron density profiles, as well as the meson mean-fields, are determined in a fully consistent way with the Coulomb interaction.

To describe inhomogeneous structures of matter, we employ Wigner-Seitz approximation: We assume a periodic structure of an ideal crystalline unit with geometrical symmetry. The shape of the unit cell is a sphere for the dimensionality $D=3$, a cylinder for 2, and a plate for 1 . By assuming a completely symmetric shape and charge-neutrality of the cell, all the calculations are reduced to that in one dimension and also we can neglect interactions among cells.

Giving an average baryon density $\rho_{B}$ and a temperature $T$, we solve field equations for mesons and the Coulomb potential determined by the density distributions of proton, neutron, and electron, $\rho_{i}(r)(i=p, n, e)$. Each fermion density distribution $\rho_{i}(r)(i=p, n, e)$ is obtained so that the chemical potential $\mu_{i}(r)=\partial \varepsilon(r) / \partial \rho_{i}(r)$ becomes uniform, where $\varepsilon(r)$ is the local energy density. The neutrino density $\rho_{v}(r)$ is always uniform. A lepton fraction $Y_{l}=\int d^{3} r\left[\rho_{e}(r)+\rho_{v}\right] / \int d^{3} r \rho_{B}$ is given and the chemical equilibrium condition $\mu_{n}+\mu_{v}=\mu_{p}+\mu_{e}$ is set into a constraint.

\section{Low-Density Nuclear Matter With and Without Neutrinos}

First, let us present in Fig. 1 the resultant structures of matter with a constant lepton fraction $Y_{l}=0.3$ at temperature $T=5 \mathrm{MeV}$. If neutrino is absent, the proton fraction becomes small and there was no chance to have pasta structures such as rod, slab, etc. $[4]^{1}$ With inclusion of neutrinos with a constraint $Y_{l}=0.3$, on the contrary, we can see all kinds of pasta structures. As we will see later, this is because the finite lepton fraction $Y_{l} \equiv Y_{e}+Y_{v}=$ const. causes the electron fraction, i.e., the proton fraction $Y_{p}$ to increase, and consequently enhances clusterization of matter.

\footnotetext{
${ }^{1}$ Whether pasta structures appear or not in the beta-equilibrium matter depends on the density dependence of the symmetry energy [9].
} 


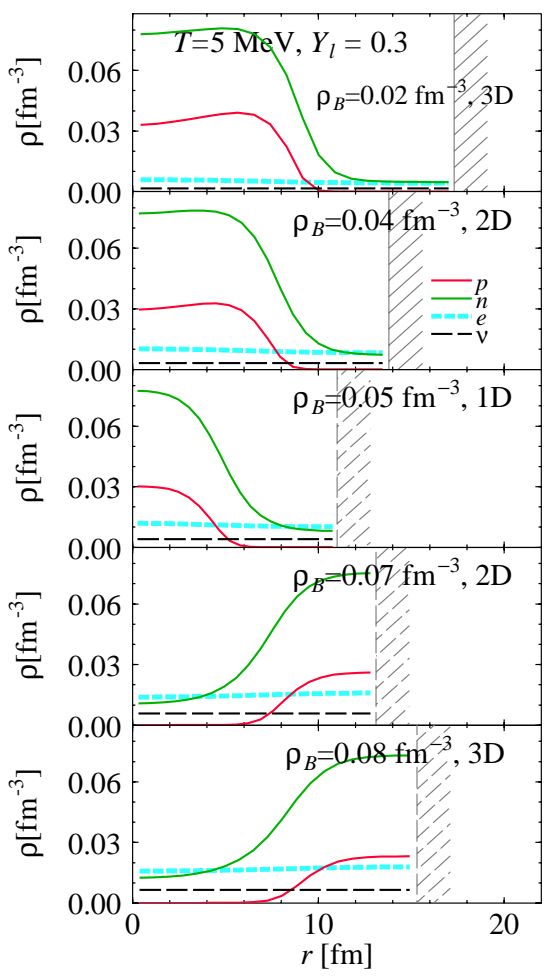

Figure 1: Density distributions in the Wigner-Seitz cells. The hatches on the right-hand side shows the cell boundaries. From the top, with increase of the baryon density, the most suitable dimensionality changes as 3,2,1,2, and 3, and the matter structure changes as spherical droplet, cylindrical rod, planar slab, cylindrical tube, and spherical bubble.

Figure 2 shows the equation of state (EOS), i.e., the pressure dependent on the baryon density. The baryon partial pressures $P_{B}$ of uniform matter (solid and dashed black lines in the upper panels) have negative gradient at lower densities, which causes mechanical instability. Therefore a firstorder phase transition occurs and there appears a mixed phase to avoid unstable regions, forming inhomogeneous pasta structures. Although the region of a largely negative gradient disappears, the overall behavior of $P_{B}$ of the pasta phases has a slightly negative gradient. However, the total presser $P_{\text {tot }}$ and its gradient are always positive as shown in the lower panel.

The proton fraction as a function of baryon density is plotted in Fig. 3. As a constraint, the lepton fraction $Y_{l}=Y_{e}+Y_{v}$ is kept constant. We can see that the resulting $Y_{p}=Y_{e}$ is about 3/4 of $Y_{l}$. One reason of this rather large ratio of $Y_{p}$ to $Y_{v}$ is that the production of an $e$ brings about another production of a $p$, i.e., higher degree of freedom compared to that of $v$ production. Another reason is that electrons and protons attract with each other via the Coulomb interaction and reduce their chemical potentials.

If there are no neutrinos in matter, the proton fraction is very small (bottom of Fig. 3). The effect of neutrino is, therefore, to increase proton fraction, which enhances the appearance of inhomogeneous structures.

As already mentioned, a constant $Y_{p}$ has been often used as a condition of supernova matter. This is justified only approximately. To see more precisely, we show the density dependence of $Y_{p}$ in Fig. 3. Interesting is that this density-dependence is different for temperatures $T=5 \mathrm{MeV}$ and $T \approx 0$, and for uniform and inhomogeneous cases. 

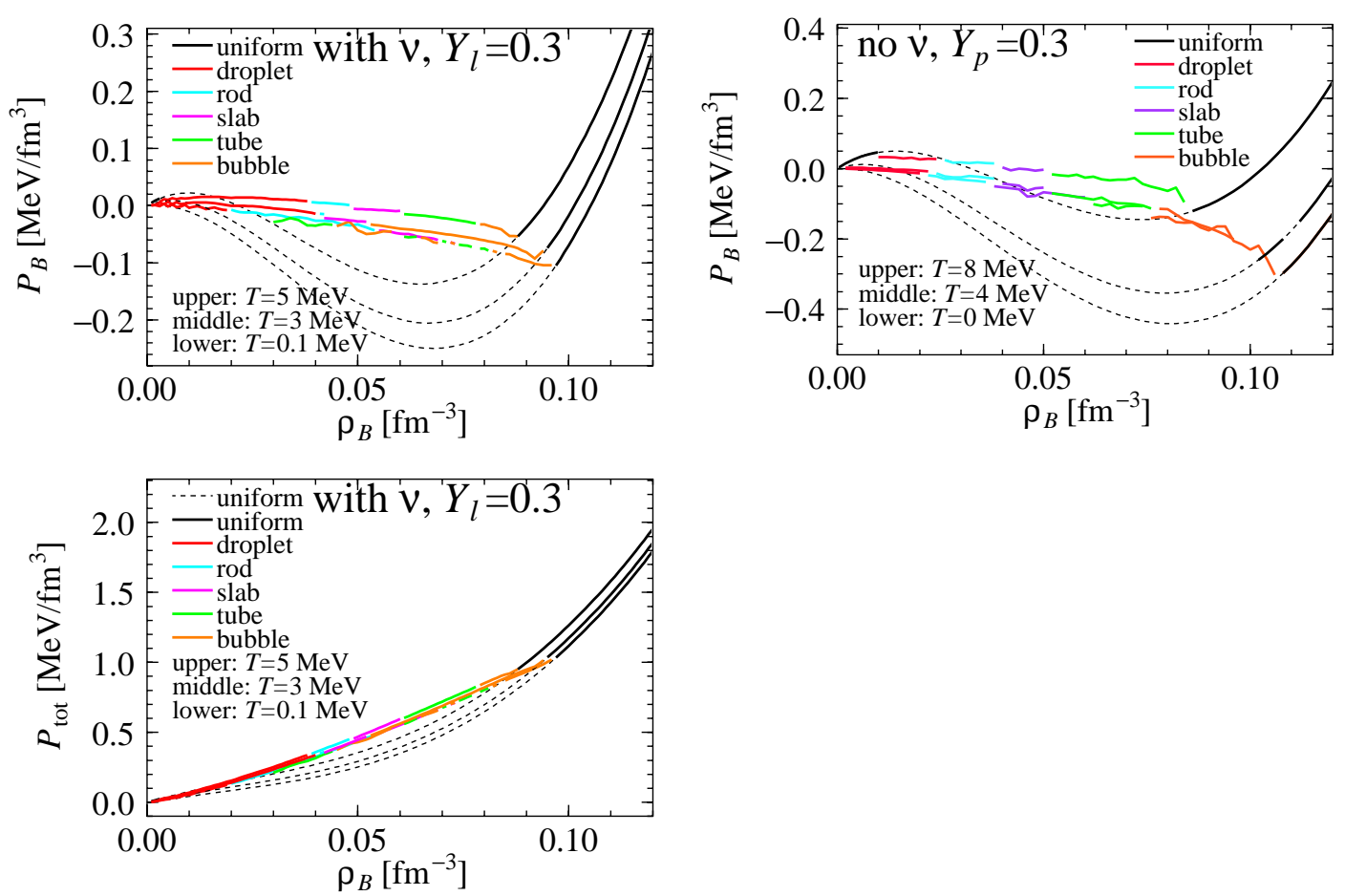

Figure 2: Upper panels show baryon partial pressures as functions of baryon density. The lepton fraction is kept constant $Y_{l}=0.3$ in the left panel, while proton fraction is constant $Y_{p}=0.3$ without inclusion of neutrino in the right panel. The lower panel shows the total pressures for $Y_{l}=0.3$. Black dashed lines show the cases where uniform matter is assumed, while colored lines show the cases where pasta structures appear.

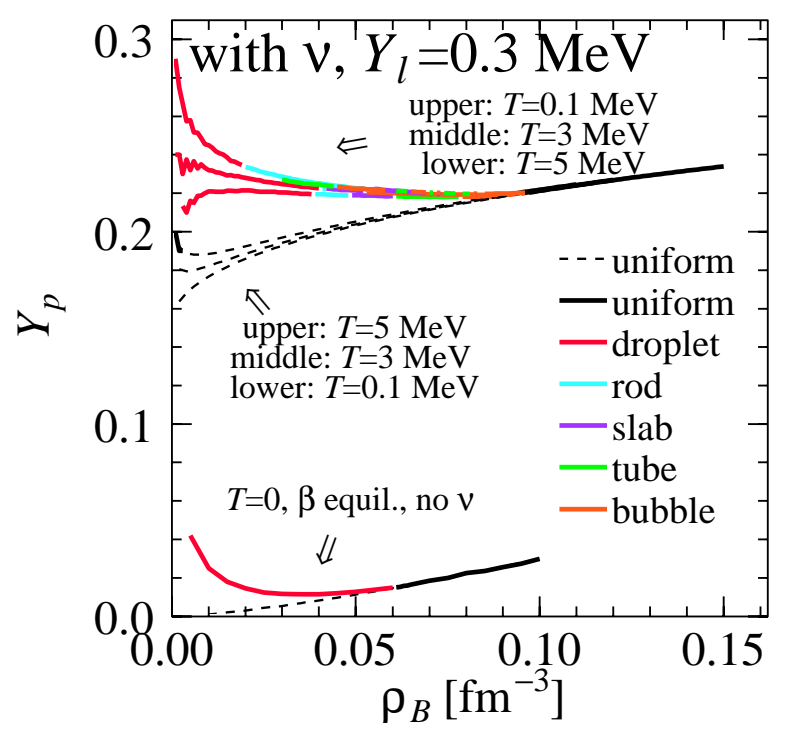

Figure 3: Density dependence of proton fraction. Lines except two lowermost ones are proton fraction $Y_{p}$ in the case of constant lepton fraction $Y_{l}=0.3$. 


\section{Summary}

We have performed relativistic mean-field calculation with Thomas-Fermi approximation for neutrino-catalyzed nuclear matter at finite temperature. We have shown results on the structure and properties of matter relevant to the core of supernovae and crust of protoneutron stars. We have seen that pasta structures appear below saturation densities, which affects the EOS. The presence of neutrinos enhances the pasta structures. It also affects the EOS of uniform matter as well as inhomogeneous matter.

Our results are consistent to the preceding studies by Ogasawara et al. and Watanabe et al. But we have extended their conclusions to more general case of matter with pasta structures. It is worth applying our calculation systematically to supernova matter so that supernova simulation can use. So far, our calculation on pasta structures assume geometrical symmetry of crystalline unit. In the future we can apply our new fully 3-dimensional calculation [10] without Wigner-Seitz approximation to neutrino-catalyzed matter at finite temperature.

\section{References}

[1] D. G. Ravenhall, C. J. Pethick, and J. R. Wilson, Phys. Rev. Lett. 27, 2066 (1983).

[2] M. Hashimoto, H. Seki, and M. Yamada, Prog. Theor. Phys. 71, 320 (1984).

[3] N. Yasutake, T. Maruyama, and T. Tatsumi, arXiv:1202.0143; Phys. Rev. D, in press.

[4] T. Maruyama, T. Tatsumi, D. N. Voskresensky, T. Tanigawa, and S. Chiba, Phys. Rev. C 72, 015802 (2005).

[5] R. Ogasawara and K. Sato, Prog. Theor. Phys. 68, 222 (1982).

[6] R. Ogasawara and K. Sato, Prog. Theor. Phys. 70, 1569 (1983).

[7] G. Watanabe, K. Iida, and K. Sato, Nucl. Phys. A 687, 512 (2001).

[8] T. Maruyama, T. Tatsumi, T. Endo, and S. Chiba, Recent Res. Devel. in Physics 7, 1 (2006).

[9] K. Oyamatsu and K. Iida, Phys. Rev. C 73, 015801 (2007).

[10] M. Okamoto, T. Maruyama, K. Yabana, and T. Tatsumi, Phys. Lett. B 713, 284 (2012). 